\title{
Eltrombopag Use for Treatment of Thrombocytopenia in a Patient with Chronic Liver Disease and Portal Vein Thrombosis: Case Report
}

\author{
Sabah E. Mohamed ${ }^{a}$ Mohamed A. Yassin ${ }^{b}$ \\ aDepartment of internal medicine, Hamad medical corporation, Doha, Qatar; ${ }^{b}$ National \\ Centre for Cancer and Research, Hamad Medical Corporation, Doha, Qatar
}

\section{Keywords}

Eltrombopag · Thrombocytopenia · Chronic liver disease $\cdot$ Thrombopoietin

\begin{abstract}
Off-label drug use refers to drug use beyond the specifications authorized for marketing [J Med Case Rep. 2014 Dec;8(1):303]. Eltrombopag is a thrombopoietin receptor agonist that has been used in treating thrombocytopenia due to chronic liver disease (CLD) as an off-label medication. Treatment of thrombocytopenia in patients with CLD constitute a real dilemma as the options are limited and some of them are invasive. However, thrombopoietin receptor agonist has been increasingly used for this purpose. Here we report a 34-year-old woman who has been diagnosed with CLD due to autoimmune hepatitis 20 years ago. Her condition was complicated with portal vein thrombosis, chronic thrombocytopenia, and variceal hemorrhage, and she has been listed as a candidate for liver transplantation. Given her high risk of bleeding, we started her on low dose of eltrombopag ( $25 \mathrm{mg}$ daily) in order to maintain a platelet level of $\geq 50 \times 10^{3} / \mu \mathrm{L}$. However, 1 year after initiation of the therapy, she developed left lower limb deep vein thrombosis.

\section{Introduction}

Chronic liver disease (CLD) is defined as "progressive destruction of the liver parenchyma over a period greater than 6 months leading to fibrosis and cirrhosis".

Thrombocytopenia is a well-known complication of CLD. It classified into moderate (less than $100 \times 10^{9} / \mathrm{L}$ ) and severe (less than $50 \times 10^{9} / \mathrm{L}$ ) thrombocytopenia [1]. The prevalence 
of thrombocytopenia in patients with liver disease ranges from $6 \%$ among patients without cirrhosis to $78 \%$ in patients with cirrhosis [2].

The pathophysiology of thrombocytopenia in these patients is multifactorial; however, the main mechanisms are platelet sequestration in the spleen and decreased production of thrombopoietin in the liver [1].

The presence of thrombocytopenia can significantly complicate routine patient care, given that most of the patients with CLD frequently undergo medical procedures for diagnosis and treatment, some of which are invasive. In addition, it limits the use of pegylated interferon therapy for patients with liver cirrhosis due to HCV infection.

Furthermore, treatment of thrombocytopenia in CLD is challenging, in that platelet transfusion can provide temporary correction of thrombocytopenia but does not ensure maintenance of hemostatic platelet levels. Several other treatment options have been used such as interventional splenic artery embolization, surgical splenectomy, and recently thrombopoietin, which regulates megakaryocyte maturation and platelet production [1].

Currently, three oral thrombopoietin agents are available to elevate platelet counts, two of these agents (avatrombopag and lusutrombopag) were approved in 2018 by the US Food and Drug Administration (FDA) for the purpose of increasing platelet counts in patients with CLD prior to an invasive procedure [2].

Although eltrombopag has gained approval from the FDA for chronic immune thrombocytopenia, its use is not recommended in patients with CLD due to the increased risk of venous thromboembolism. However, it has been used in several studies as an off-label medication for treatment of thrombocytopenia in CLD [2-6].

In this case report, we are highlighting the challenge of treating thrombocytopenia in a patient with CLD complicated by variceal hemorrhage and portal vein thrombosis.

\section{Case Presentation}

We report a 34-year-old Palestinian woman who is known to have SLE and CLD due to autoimmune hepatitis, both diagnosed 20 years ago. As her CLD progressed, she developed advance liver cirrhosis with portal hypertension and massive splenomegaly. In 2012, she had upper gastro-intestinal bleeding, esophagogastroduodenoscopy (OGD) revealed esophageal varices and banding was done. One year later, she had a second episode of variceal hemorrhage. Since that time, she was under regular endoscopic surveillance with frequent banding to control varices and prevent bleeding recurrence. However, despite of supportive therapy, she continued to have recurrent hospital admissions due to decompensation. Moreover, her condition complicated by portal vein thrombosis and chronic thrombocytopenia with fluctuation of platelet count which reached a nadir of $17 \times 10^{3} / \mu \mathrm{L}\left(150 \times 10^{3} / \mu \mathrm{L}\right.$ to $\left.400 \times 10^{3} / \mu \mathrm{L}\right)$. In 2016 , the patient was referred to a liver transplant clinic; at that time, her MELD score was 18, her Child-Pugh classification was B, and she was listed as a candidate for liver transplantation.

Given her high risk of variceal hemorrhage in addition to the use of anticoagulation for treatment of portal vein thrombosis, and the plan for a liver transplant, it was judicious to maintain platelet level of $\geq 50 \times 10^{3} / \mu \mathrm{L}$. So, the decision to start thrombopoietin was made. In 2017 , the patient was started on low dose of eltrombopag $25 \mathrm{mg}$ daily. The platelet count at the beginning of the treatment was $20 \times 10^{3} / \mu \mathrm{L}$; 4 weeks later, it increased to $58 \times 10^{3} / \mu \mathrm{L}$ and it was maintained at $\geq 50 \times 10^{3} / \mu \mathrm{L}$ (but less than $100 \times 10^{3} / \mu \mathrm{L}$ ) in the subsequent followup on the same dose.

In 2018, the patient presented to the emergency department with left lower limb pain and swelling for one week. Doppler ultrasound of the left leg showed thrombosis of the distal posterior tibial vein with mild subcutaneous edema in the left calf. Platelet count at that time 
was $66 \times 10^{3} / \mu \mathrm{L}$. However, although this incidence was attributed to the use of eltrombopag, after discussion of the benefits and the risks with the patient, the decision to continue eltrombopag was made.

\section{Discussion}

Eltrombopag is an orally bioavailable, small-molecule, thrombopoietin receptor agonist that selectively binds to thrombopoietin receptors on megakaryocyte precursors and megakaryocytes leading to increased platelet production. It has been approved for treatment of thrombocytopenia due to immune thrombocytopenic purpura. Also, it has been used in patients with chronic HCV infection to allow for the initiation and maintenance of peginterferon-based therapy [6]. However, studies regarding its safety and efficacy in treating thrombocytopenia in CLD due to autoimmune hepatitis are still deficient.

In our patient, eltrombopag has been used at low dose, yet it has been effective in maintaining platelets above the critical level.

Kawaguchi et al. [5] showed in their study that a daily dose of $25 \mathrm{mg}$ of eltrombopag was effective in raising platelet count to $>50 \times 10^{3} / \mu \mathrm{L}$ in Japanese patients with CLD in 2 weeks. Nevertheless, our patient needed more than 3 weeks to reach the target platelet level. This can be attributed to inter-ethnic differences in the pharmacokinetics of eltrombopag, which have been reported in previous studies [5].

The decision to start eltrombopag in our patient who is known to have portal vein thrombosis was really challenging. However, the critically low platelet level and being on anticoagulation for treatment of portal vein thrombosis, in addition to the patient history of recurrent variceal haemorrhage, which carries high risk of mortality, and the fact that eltrombopag was the only option available were enough compelling reasons to start this therapy as it was a life-saving treatment for this patient.

Nonetheless, despite using low dose of eltrombopag and maintaining the platelet level at around $50 \times 10^{3} / \mu \mathrm{L}$ to avoid risk of thrombotic events, our patient developed lower limb deep vein thrombosis one year after starting eltrombopag. One study of eltrombopag use in patients with cirrhosis and thrombocytopenia showed a positive correlation between platelet count of $\geq 200 \times 10^{3} / \mu \mathrm{L}$ and incidence of portal vein thrombosis. However, they used a higher dose of eltrombopag (75 mg daily) for 14 days [4]. Up to date, there is no study conducted regarding the long-term use of eltrombopag in patients with CLD. Hence, more studies need to be carried out in order to explore this area.

\section{Conclusion}

Eltrombopag was effective in ameliorating thrombocytopenia in a patient with CLD due to autoimmune hepatitis; however, the risk of thrombosis still exists even with a small dose and a platelet level below $100 \times 10^{3} / \mu \mathrm{L}$. Thus, the decision to start and to continue eltrombopag should be individualized according to the patient's condition.

\section{Acknowledgement}

We would like to thank the Internal Medicine Residency Program at Hamad Medical Corporation, especially Dr. Dabia Al Mohanadi and Dr. Ahmed Al Mohammed, for their continuous support. Also, we thank Qatar National Library for the resources and opportunity. 


\section{Case Reports in Oncology}

Mohamed/Yassin: Eltrombopag Use in Chronic Liver Disease

\section{Statement of Ethics}

This case was conducted in accordance with the World Medical Associated Declaration of Helsinki. Informed Written consent was taken from the patient to publish her case.

\section{Conflict of Interest Statement}

The authors have no conflict of interest to declare.

\section{Funding Sources}

This work is financially supported by the Qatar National Library, Doha, Qatar.

\section{Author Contributions}

Dr. Sabah E. Mohamed: literature review and manuscript writing. Dr. Mohamed A. Yassin: literature review and manuscript editing.

\section{References}

1 Hayashi H, Beppu T, Shirabe K, Maehara Y, Baba H. Management of thrombocytopenia due to liver cirrhosis: a review. World J Gastroenterol. 2014 Mar 14;20(10):2595-605.

2 Miller JB, Figueroa EJ, Haug RM, Shah NL. Thrombocytopenia in chronic liver disease and the role of thrombopoietin agonists. Gastroenterol Hepatol (N Y). 2019 Jun;15(6):326-32.

3 Al-Jafar HA, Al-Khaldi J, Alduaij A, Al-Banwan K. Severe thrombocytopenia in a patient with hepatitis C treated with eltrombopag from off-label drug use to on-label drug use: a case report. J Med Case Rep. 2014 Dec;8(1): 303.

4 Afdhal NH, Giannini EG, Tayyab G, Mohsin A, Lee JW, Andriulli A, et al. Eltrombopag before procedures in patients with cirrhosis and thrombocytopenia. N Engl J Med. 2012 Aug 23;367(8):716-24.

5 Kawaguchi T, Komori A, Seike M, Fujiyama S, Watanabe H, Tanaka M, et al. Efficacy and safety of eltrombopag in Japanese patients with chronic liver disease and thrombocytopenia: a randomized, open-label, phase II study. J Gastroenterol. 2012 Dec 1;47(12):1342-51.

6 Afdhal NH, Dusheiko GM, Giannini EG, Chen PJ, Han KH, Mohsin A, et al. Eltrombopag increases platelet numbers in thrombocytopenic patients with HCV infection and cirrhosis, allowing for effective antiviral therapy. Gastroenterology. 2014 Feb 1;146(2):442-e1. 